\title{
Bilateral Vibrotactile Feedback Patterns for Accurate Lateralization in Hearing Instrument Body Area Networks
}

\author{
B.Tessendorf, D.Roggen, \\ M.Spuhler, T.Stiefmeier \\ G. Tröster \\ Wearable Computing Lab., \\ ETH Zurich, Gloriastr. 35 \\ 8092 Zurich, Switzerland \\ \{lastname\}@ife.ee.ethz.ch
}

\author{
T.Grämer \\ Laboratory for Experimental \\ Audiology \\ University Hospital \\ Frauenklinikstr. 24 \\ 8091 Zurich, Switzerland \\ Tobias.Graemer@usz.ch
}

\author{
P.Derleth, M.Feilner \\ Phonak AG \\ Laubisrütistrasse 28 \\ 8712 Stäfa, Switzerland \\ \{firstname.lastname\}@phonak.com
}

\begin{abstract}
Hearing Instruments (HIs) have emerged as true body area networks, so called HI-BANs. Besides streaming audio data they connect wirelessly to accessories such as remote controls and Bluetooth devices. Multimodal sensor data from a HI-BAN is a way to adapt the HI behavior to the user's current hearing situation. As a potential future HI-BAN component we investigate bilateral vibrotactile feedback to support localization of sound sources. As a foundation for integrating vibrotactile cues we investigate which kind of feedback and vibration patterns are most suitable. We implemented two approaches for encoding lateral target angles: Continuous Guidance Feedback (CGF) and 6 variants with evolving complexity of Quantized Absolute Heading (QAH). In a user study with 16 normal hearing participants $(7 \mathrm{~m}, 9 \mathrm{f}$, age 23-61) we evaluate lateralization error and user response time. For QAH results show a trade off between the minimal quantization error due to the encoding and the number of user errors due to misinterpretation of presented patterns. Moreover, results show a trade off between response time and minimum lateralization error. Choosing the most suitable bilateral vibrotactile encoding schemes is applicationspecific: For QAH a minimal average lateralization error of $27^{\circ}\left(\sigma=22^{\circ}\right)$ was achieved with eight $45^{\circ}$-segments and an average user response time of $1600 \mathrm{~ms}(\sigma=545 \mathrm{~ms})$. A minimal average user response time of $900 \mathrm{~ms}(\sigma=325 \mathrm{~ms})$ was achieved with four $45^{\circ}$-segments and an average lateralization error of $43^{\circ}\left(\sigma=29^{\circ}\right)$. CGF guides the user within a given tolerance margin to the target at the cost of higher response time. We find that for complex encoding schemes the overall performance is person-specific.
\end{abstract}

\section{Categories and Subject Descriptors}

H.5.2 [User Interfaces]: Haptic I/O

Permission to make digital or hard copies of all or part of this work for personal or classroom use is granted without fee provided that copies are not made or distributed for profit or commercial advantage and that copies bear this notice and the full citation on the first page. To copy otherwise, to republish, to post on servers or to redistribute to lists, requires prior specific permission and/or a fee.

BODYNETS 2011, November 07-08, Beijing, People's Republic of China Copyright (C) 2012 ICST 978-1-936968-29-9

DOI 10.4108/icst.bodynets.2011.246910

\section{General Terms}

Hearing Instrument, Vibrotactile Feedback, User Study

\section{INTRODUCTION}

Recent studies have shown that hearing impairment is increasingly affecting people worldwide, especially in developed countries $[6,13]$. In particular, localization of sound sources is an essential ability of the human auditory perception. It enables the listener to turn towards an interesting sound source to maximize intelligibility or to identify approaching vehicles to be safe in traffic situations. It is challenging for listeners with a strong asymmetric hearing loss to localize sound sources in the horizontal plane, also referred to as lateralization. The large group of affected people includes unilateral deaf, unilaterally implanted cochlear implants (CIs), and also listeners with bilaterally implanted CIs as well as listeners with a central hearing loss high up in the auditory pathway. Moreover, the human lateralization relies on cues that can end up distorted due to signal processing in HIs. This also leads to decreased lateralization performance [8].

People that experience difficulties with lateralization often wear HIs or CIs and these devices are in the progress of becoming true multimodal interaction interfaces. Therefore, we investigate how to address lateralization difficulties by integrating alternate feedback modalities into HIs or CIs. HIs communicate with a variety of other accessories such as remote controls, Bluetooth, or FM devices as well as the user's smart phone to form wireless networks, so-called hearing instrument body area networks (HI-BANs) [2]. The rising trend to higher-end HIs motivates and supports our investigation of additional sensor and actuator modalities for HIs that may eventually be included within the HI itself. In particular, the integration of vibrotactile feedback into HIs or CIs is a promising approach to support the hearing impaired with lateralization or at least to provide the side of the most prominent sound source around.

To address the question "how accurate and fast can listeners localise target angles in the lateral plane through bilateral vibrotactile feedback at the ears?" we designed a continuous guidance feedback and 6 bilateral vibrotactile encoding schemes with evolving complexity optimized for encoding the lateral target angle in the complete $360^{\circ}$-range using bilateral vibrotactile feedback behind the ears. 


\section{RELATED WORK}

A pair of glasses enhanced with 4 vibrators and 3 microphones with the purpose to locate sound sources for visually and hearing impaired people was presented in [3]. The approach doesn't focus on integration into HIs but the user is required to wear special goggles.

Research regarding tactile sensitivity measure for the head is presented in [9]. They found frequencies around $32 \mathrm{~Hz}$ to be optimal for perception and concluded that the part around the ears are one of the most sensitive head regions for vibrotactile stimulation. The region behind the ears at the mastoid bone is one of the most sensitive head regions for vibrotactile stimulation [9].

In [18] the authors present a device to be placed inside the ear channel to transduce sound intro vibration. The device has two active states with two different vibration intensity levels: one for high and one for low frequency sound.

A pedestrian navigation system using vibration of mobile phones is shown in [11]. The running direction is encoded in the length of vibration and the distance in the pause time between two pulses. A further navigation system is presented in [14]. They use a vibrotactile waist belt to provide vibrotactile feedback at 8 locations to allow hands-free navigation in unfamiliar places for blind people. A direction is represented by a one-to-one mapping of the respective tactor on the waist belt facing this direction.

Audio feedback can be used, as e.g. in [7] where the authors propose a portable, self-contained system that allows visually impaired people to travel through an unfamiliar environment. The information about the navigation is conveyed by earphones to the user. The disadvantage of this overlaying approach is potential interference of the computer voice with the actual real life sound.

A cell phone based approach is presented in [1]. The authors investigate to use one vibrator in a mobile-phone device and let the user use it as a vibrating pointing device, e.g. to find a friend in a crowd. Their approach could be adapted to be based on head movement instead of pointing with a mobile phone to increase intuitiveness and remove the requirement that the user must hold a device in the hand to benefit.

In [15] the authors propose to integrate sensors for body and eye movement into HI-BANs as they were found to improve $\mathrm{HI}$ performance, automatic hearing program selection in particular. As this kind of HI-BAN functionality improves the context-awareness of HIs it can help to decide when providing lateralization feedback to the user is adequate.

\section{BILATERAL VIBROTACTILE ENCOD- ING OF LATERAL TARGET ANGLES}

\subsection{Experimental Setup}

We designed a bilateral vibrotactile feedback system for lateralization to investigate which kind of feedback and vibration patterns are optimal to provide support for lateralization [16]. We evaluated a wide range of vibrators and selected coin-shaped vibrators with a diameter of $10 \mathrm{~mm}$ (310103 Vibration Motor $2.7 \mathrm{~mm}$ Button Type from Precision Microdrives) that have been developed for vibratotactile feedback in handheld applications. We emulate the integration of vibrotactile feedback into HIs by enhancing a pair of goggles with vibrators at the sidepieces as depicted in Fig-
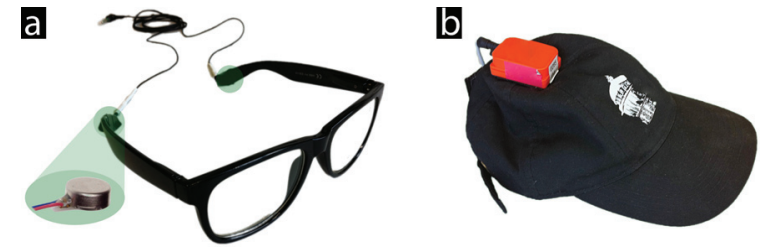

Figure 2: (a) A pair of glasses was enhanced with miniaturized vibration motors at each of the sidepieces. The glasses serve just as a mounting mechanism for the vibrators to emulate integration into HIs. (b) To track the user's head movements for CGF we use a XSens MT9 sensor on a baseball cap.

ure 2. Instead of glasses we could also enhance headphones as we use them just as an unobtrusive mechanism to press the vibration motors on the mastoid bone to effectively conduct vibrations. We control and power the vibration motors with an USB Bit Whacker [12] module combined with a motor driver chip (TI ULN2803A). Via a serial interface of a desktop computer we use pulse width modulation (PWM) to flexibly control the vibrators' intensity to implement a variety of different vibration patterns. To track the user's head angle relative to the target we attached an inertial measurement unit (XSens MT9 IMU, accuracy $\pm 0.5^{\circ}$ ) on a baseball cap as depicted in Figure 2 to track the user's head yaw angle.

\subsection{Quantized Absolute Heading}

We define vibrotactile encoding schemes as sets of patterns to encode lateral target angles. Based on existing guidelines $[4,5,10,17]$ we designed a set of bilateral vibrotactile encoding schemes specifically suitable for lateralization of target angles in the complete $360^{\circ}$-range. Table 1 gives an overview of the bilateral vibrotactile encoding schemes designed for encoding lateralization information. Considering a potential integration into HIs we focus on bilateral vibrotactile stimulation, i.e. presenting vibrotactile cues behind each user's ear. We further focus on intuitive vibrotactile encoding schemes that can be learned within a few minutes. Our proposed patterns range from basic encodings with low angular resolution to more complex ones with finer angular resolution. Figure 1 illustrates encoding schemes S1-S6. We divide the lateral $360^{\circ}$-range into four base segments: front (red), left (green), right (purple) and back (black), each with a size of $90^{\circ}$. The schemes S2-S4 consecutively extend S1. They are composed of various combinations of the 4 base

\begin{tabular}{cccc}
\hline Name & \#Segments & Segment Size & \#Intensities \\
\hline S1-S4 & $\{4,6,8,12\}$ & $\left\{90^{\circ}, 60^{\circ}, 45^{\circ}, 40^{\circ}\right\}$ & 1 \\
S5-S6 & $\{8,12\}$ & $\left\{45^{\circ}, 30^{\circ}\right\}$ & 3 \\
\hline CGF & continuous & - & 2 \\
\hline
\end{tabular}

Table 1: Overview of bilateral vibrotactile feedback approaches delivered behind the ear for encoding lateralization information. For QAH (S1-S6) and CGF the number of vibration intensities and segments with their corresponding sizes are given. 

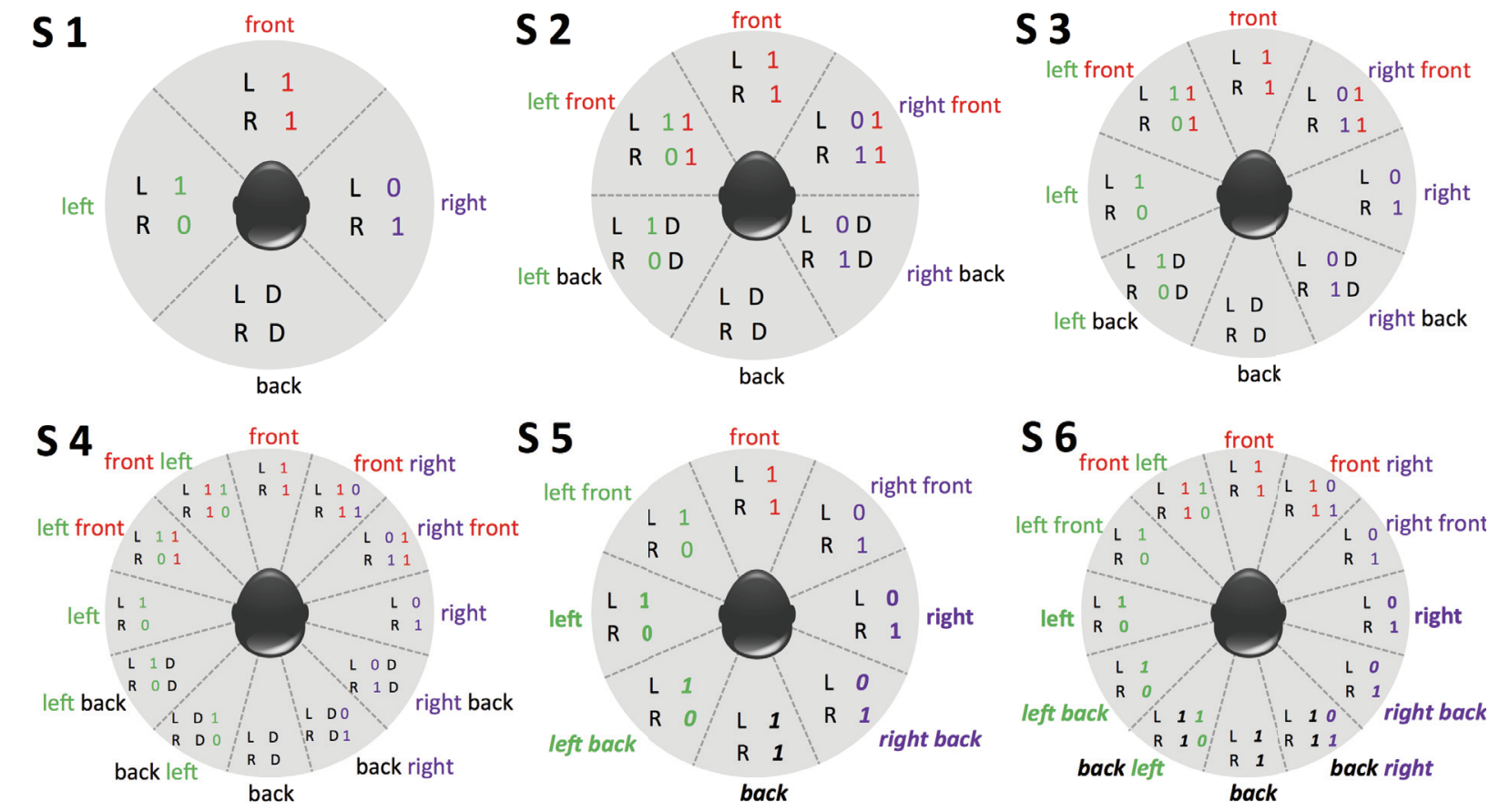

Figure 1: QAH partitioning and encoding of the lateral $360^{\circ}$-range into $4,6,8$ and 12 segments. ' $L$ ' stands for left, ' $R$ ' for right side. ' 0 ' and ' 1 ' mean vibration off and on, respectively. ' $D$ ' represents a double click sequence. For S5-S6 three intensitiy levels are used: high vibration (italic bold), medium (bold), and low (normal). For schemes S1-S4 intensity is always high (formatted as normal font for clarity).

elements and represent a refinement of the preceding ones. The segment size is reduced to $60^{\circ}, 45^{\circ}$ and $30^{\circ}$, respectively. This evolving procedure, by means of composing more complex tactile messages of well-known meaningful component was proposed in [17]. It makes learning the vibrotactile encoding schemes easier and more intuitive for the user. As disclosed in [4] it is possible to encode information using different vibration intensities. This has been considered in another encoding approach illustrated in S5 and S6. In [17] the authors recommend a maximum number of four different intensity levels. We used up to three vibration intensities for schemes S5-S6 considering that the vibration motors are not directly attached on the skin but on the sidepieces of the glasses. Schemes S5 and S6 are variants of schemes S3 and $\mathrm{S} 4$, introducing three different vibration intensities: low (PWM duty cycle of 0.1), medium (PWM duty cycle of 0.5 ) and high (PWM duty cycle of 1 ). The low intensities are used for target angles in front of the user. The high intensities are used to emphasize vibrotactile feedback for sources coming from the back of the user that usually appear more unexpectedly.

\subsection{Continuous Guidance Feedback}

Schemes S1-S6 that inform the user about the target location. In contrast, continuous guidance feedback (CGF) guides the user until the user has successfully turned the head to the target. The user's head yaw angle is measured with an inertial measurement unit (IMU) to track the current angle to the target. To eliminate the need of the head tracker for CGF in later $\mathrm{HI}$ application, the tracking could be performed using sound localization with a microphone

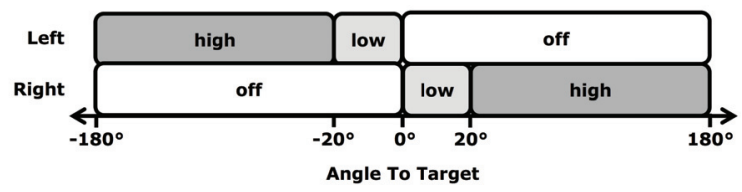

Figure 3: CGF: Continuous encoding of target angles based on the user's current angle to target. 'Low' and 'high' stand for vibration at half and full intensity, respectively.

array. CGF tells the user to turn the head to the target by vibrating on the corresponding side of the target. This vibrotactile feedback is provided continuously based on the current angle to the target. The encoding is performed with two intensity levels as illustrated in Figure 3: If the target is near, meaning in the range of $20^{\circ}$, the vibration intensity is halved. The vibrotactile feedback stops when the user has moved the head to the target within a tolerance of $5^{\circ}$.

\section{USER STUDY}

We recruited 16 participants ( 7 males, 9 females, age 2361 ). In this empirical study we recruited normal hearing subjects. In our discussions with a hearing aid manufacturer and a university hospital in charge of hearing impairment pa- 


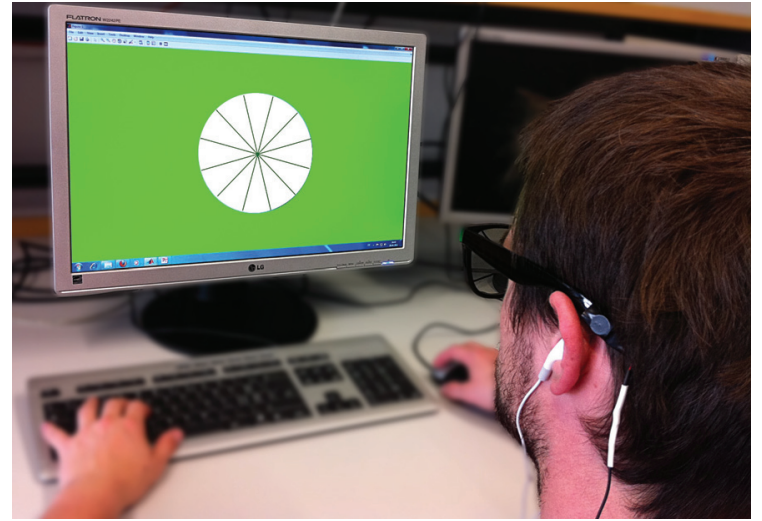

Figure 4: A participant wearing the vibrotactile glasses during the experiment and indicating the perceived target angle by clicking with a mouse into the corresponding segment on the screen.

tients ${ }^{1}$, we found that we can take the results obtained with these participants as a starting point for hearing impaired.

A set of 60 angles was pseudo-randomly generated with a continuous values range between $0^{\circ}$ and $360^{\circ}$. We used a number 60 angles to aim for an even covering of the values range to maintain a reasonable overall duration of the experiment. The set of stimuli angles were the same for all participants to allow for consistent comparison. Each target angle was encoded with a vibrotactile pattern and then presented to the participant. The presentation of the consecutive patterns took place with random pauses between 1 and 3 seconds to not let the user get used to time slots. For each participant the same stimulus vector was taken. Participants were instructed to localize as fast and accurate as possible. As an incentive for participation the best participant won a price. For QAH they indicate the perceived target angle by clicking with a mouse anywhere into the segment of a circle displayed on the computer screen as shown in Figure 4. The middle value of the corresponding segment is used to calculate the deviation to the target angle. The time between start of the presentation of the vibrotactile pattern and the user response is stopped. For CGF the user response time is automatically stopped as soon as the user has turned his head to the target within a given $5^{\circ}$ tolerance margin. As a baseline we also measured the user response time without vibrotactile feedback but using visual stimuli only. The target angle was displayed as a large marker in the corresponding position circle on the computer screen. This response time serves as a baseline to interpret the values of the user response times from vibrotactile feedback. During the experiment the participants were listening with earphones to wide-band music to make sure that they are not influenced from any sound from the vibrators potentially produced during operation. The pair of glasses fitted all participants well. Before each experiment run with a different

${ }^{1}$ This work was carried out in collaboration with a hearing instrument company and an university hospital. In a planned extended study we will investigate combined acoustic and vibrotactile feedback to further evaluate the benefit of the system for hearing impaired listeners.

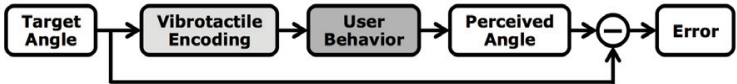

Figure 5: Calculation of the resulting lateral angle deviation, influenced by the vibrotactile encoding due to quantization and user errors due to misinterpretation of patterns.

vibrotactile encoding scheme, the participants completed a short tutorial supported by the person who supervised the user study to become familiar with the system and vibrotactile encoding schemes. Finally the participants completed a questionnaire together with the person who supervised the user study. In a common software framework we unify the presentation of the vibrotactile stimuli for the virtual target angle, retrieving user feedback from a GUI, control of the vibrators and head tracker and measurement of the user response time and angular error.

\section{PERFORMANCE EVALUATION}

We evaluated vibrotactile encoding schemes considering lateralization performance and user response time.

Lateralization Angle Error Figure 5 illustrates the flow of information: The target angle is encoded into a vibrotactile pattern and presented to the user. The user interprets the vibrotactile pattern and indicates the perceived angle. The resulting error is influenced by both the vibrotactile encoding due to quantization and user errors due to misinterpretation of patterns. We investigate both influencing factors separately as well as the resulting combined influence on the overall performance. As an error measure for the angular deviation we use root mean square (RMS) to consider greater deviation with greater error values. The performance for CGF is intrinsically guaranteed to the given tolerance of $5^{\circ}$ because the feedback continues until the target is found. Therefore also no user errors occur with this scheme. Figure 6 shows the user error due to misinterpretation of the presented pattern for each participant as share of the overall number of presented stimuli. Figure 7 shows user response time for each participant and on average over all participants. For the CGF corresponds we define the average duration of the pattern as user response time in a wider sense. The user needs more time to locate far targets than near targets. On average the users needed a time of $902 \mathrm{~ms}$ ( $\sigma=161 \mathrm{~ms}$ ) to operate the GUI with visual stimuli only as described in section 4 as shown with the bars for the case 'visual' in Figure 7. Figure 8 shows the RMS lateralization error for each participant and on average over all participants.

User Response Time We measure the user response time starting from the initiation of the vibrotactile feedback until the mouse click in the GUI (for CGF until the user reached the target angle by moving the head). This includes the motor start delay (we neglect it because it is in the range of $60 \mathrm{~ms}$ according to data sheets), the pattern duration, the user response time and the time to operate the GUI. To consider the time to operate the GUI, we also measured user response time based on visual stimuli only. No direct user feedback is required for the CGF approach. 


\section{USER QUESTIONNAIRE}

The participants assessed 24 statements about intuitiveness of the different encoding schemes, the source of error for confusing patterns and the comfort of the vibrotactile glasses. A continuous rating scale from 0 ('not true') to 10 ('very true') has been used and the participants had to put a mark on the scale, depending how true the statement was. At the end of the questionnaire the study participants had the opportunity to answer some open questions about improvements and general comments. As a result of the rating scale the CGF approach was evaluated as the most intuitive scheme, before the patterns $\mathrm{S} 1-\mathrm{S} 4$. The schemes S5 and S6 that featured three different vibration intensities were rated as the least intuitive ones. Most participants stated to have remembered the coding schemes during the test phase after a few minutes of memorizing. As the main cause of error, the participants answered that some vibration patterns - especially the three intensities in S5 and S6, i.e. right front, right and right back- were hard to distinguish. But overall the intensity of the vibration has been felt as appropriate and comfortable. The feedback we got concerning the comfort of the vibrating glasses was without exception positive: All of the participants stated to not feel tense, physically different or strange wearing the device and that the vibration wasn't perceived as painful or disturbing even after one hour of wearing it.

\section{DISCUSSION}

\subsection{Lateralization Performance and User re- sponse time}

Results show a trade off between user response time and minimum angular lateralization error. Choosing the most suitable bilateral vibrotactile encoding schemes depends on the requirements of the application:

- To minimize the RMS lateralization error, scheme S3 is the most suitable scheme out of schemes S1-S5. It encodes 8 segments of size $45^{\circ}$ and achieves an average RMS lateralization error of $27^{\circ}\left(\sigma=22^{\circ}\right)$ an average user response time of $1600 \mathrm{~ms}(\sigma=545 \mathrm{~ms})$. For participants P11, P13 and P15 scheme S4 that encodes 12 segments of size $30^{\circ}$, and for participants P8, P10 and P14 one of the schemes S5 or S6 that encodes three vibration intensities show the lowest error. With the CGF approach maximum lateralization performance was achieved by guiding the user to the given $5^{\circ}$ tolerance margin to the target. However, CGF shows an increased average user response time of $3140 \mathrm{~ms}(\sigma=2468 \mathrm{~ms})$ and a four times higher average energy budget than scheme S3.

- To minimize response time, e.g. alerting in emergency situations like an approaching car from behind, vibrotactile encoding scheme $\mathrm{S} 1$ is most suitable. It encodes 4 segments of size $90^{\circ}$ and achieves an average user response time of $900 \mathrm{~ms}(\sigma=325 \mathrm{~ms})$ with an average RMS lateralization error of $43^{\circ}\left(\sigma=29^{\circ}\right)$.

The lateralization performance required depends on the application: E.g. for emergency situations, it might be desired to just inform about the side a potential harm is coming from to let the user move out of the way, as fast as possible by minimizing user response time. Another example application is resolving front-back confusions that are a well-known issue in lateralization [8] by indicating the half space of a sound source. For different applications the goal might be a maximization of lateral accuracy, e.g. to find targets that are far away best possible.

The resulting RMS lateralization error is influenced by both the vibrotactile encoding due to quantization and user errors due to misinterpretation of patterns as illustrated in Figure 5. The influence of the two error sources are shown with Figures 6 and 8. An example to illustrate the influence the quantization effects is the case of participant P9 who made almost now user errors. The corresponding RMS lateralization errors still show a lower bound due to quantization during vibrotactile encoding. This is due to the quantization of the lateral $360^{\circ}$-range into segments and the fact, that target angles do occur continuously inside the segments.

For QAH encoding schemes results show a trade off between the minimal quantization error due to vibrotactile encoding and the average number of user errors due to misinterpretation of presented patterns. The simpler the schemes are concerning the number of encoded segments, the lower are the user error and response time, but the higher the induced quantization error due to vibrotactile encoding. On the other hand, reducing the quantization error with encodings of smaller segments induced a higher number of user errors and user response time. The smaller the segments, the less impact has the type of user error that confuses an adjacent segment on the resulting RMS lateralization error. For the complex encoding schemes the overall performance of the vibrotactile feedback for lateralization is person-specific. E.g., participant P9 would maybe cope with more complex schemes, however other participants didn't show as low user error rates for all encoding schemes. The standard deviation for the depicted in Figure 6 as errorbars for the average case is an indicator to characterize how person-specific the performance turns out for each encoding scheme. Figure 6 shows the relative number of times the user confused a pattern. The values are not directly comparable between schemes, because the difficulty to recognize patterns is different for the different vibrotactile encoding schemes. The outlier for participant P14 and scheme S4 in Figure 6 was explained by the corresponding participant by not having remembered the scheme.

Figure 7 shows the time for the baseline response time based on visual stimuli online. The standard deviation when averaging over all participants seems sufficiently low to skip the measurement of the baseline in further experiments and to assume a common constant offset for all participants. Average response times for scheme $\mathrm{S} 1$ are in the range of and for some participants even below the baseline. This confirms previous research that the haptic sense can lead to quicker response times than for the visual baseline. CGF shows highest response time, because it does not inform the user, but provides continuous feedback until the target is found. The time for lateralization increases with the initial distance of the target. To compare schemes S1-S6 and CGF a main difference needs to be considered: For schemes S1-S6 the user is just informed about the target location and still needs to move the head into the correct direction if desired. 


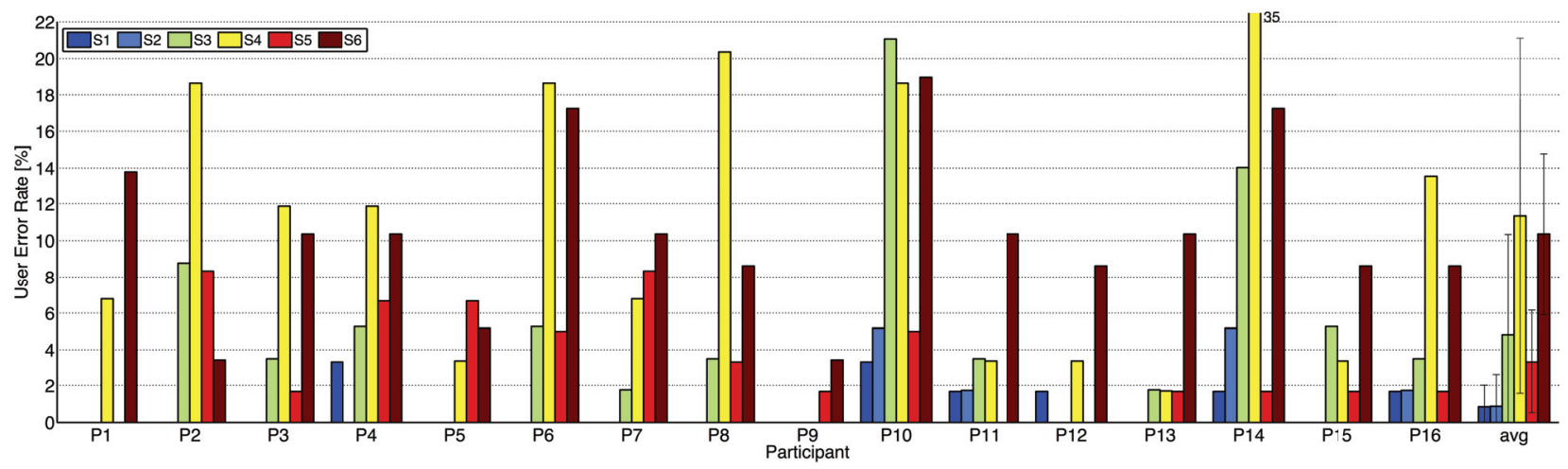

Figure 6: User error rate: The share of user errors due to misinterpretation of patterns for each participant. Also average values and the standard deviation across all participants are given. All confusions are counted as one error. For CGF this measure is not applicable.

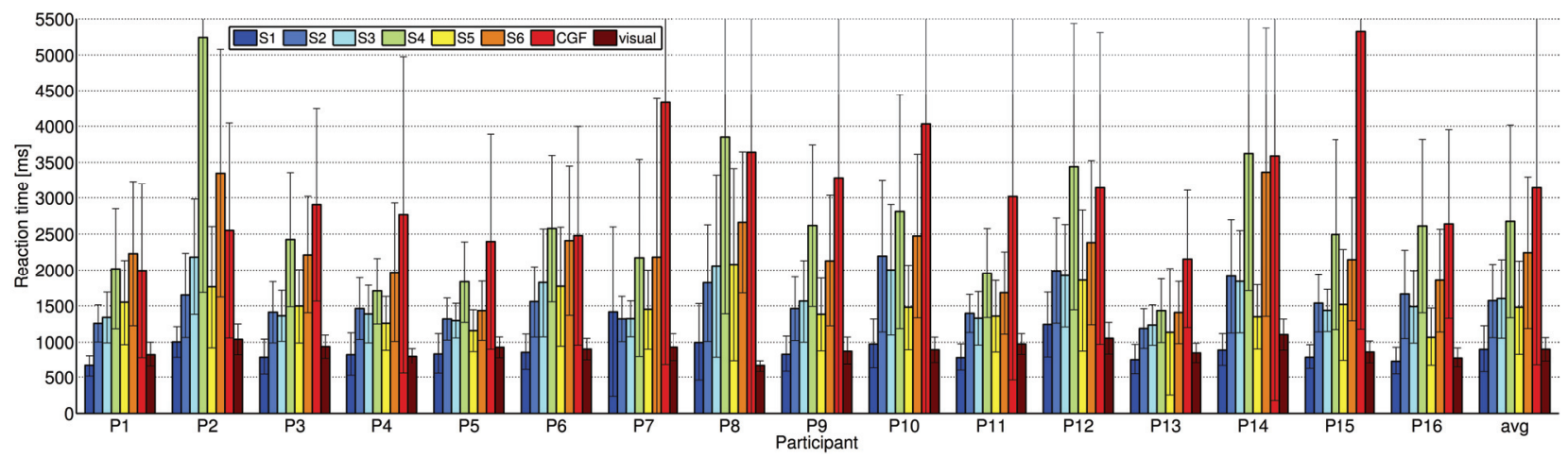

Figure 7: User response time for each participant and standard deviation. Also average values and the standard deviation across all participants are given. Also the baseline based on visual stimuli is showing. For CGF the response time correspond to the time needed to find the target.

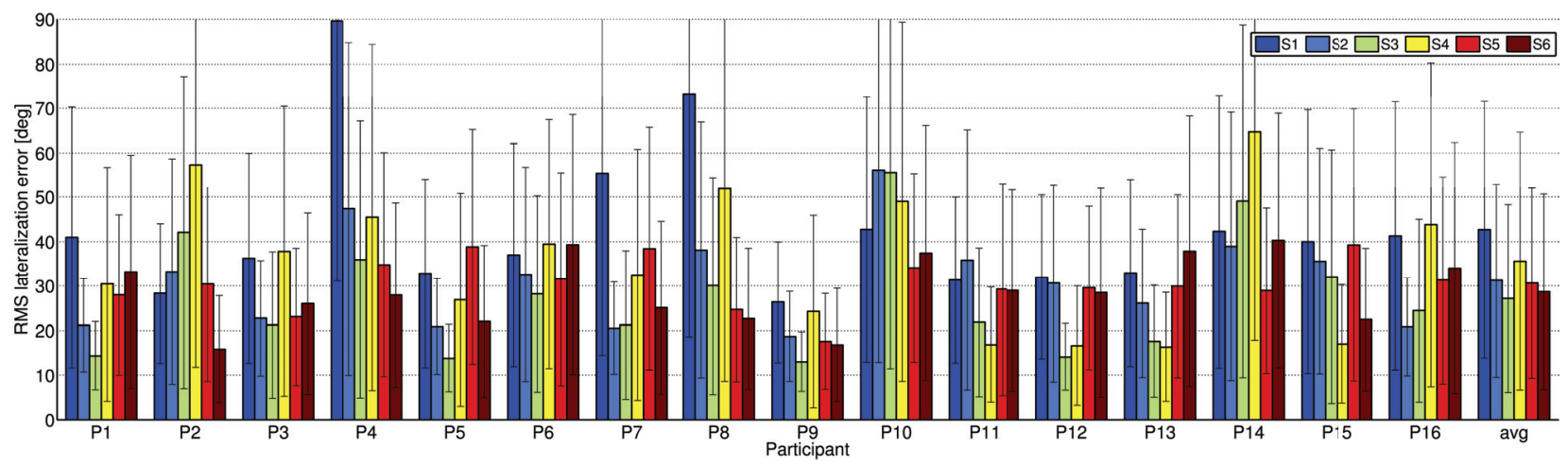

Figure 8: RMS lateralization error: This measure including the quantization effect due to vibrotactile encoding where applicable and the effect of the user error due to misinterpretation of patterns. Also average values and the standard deviation across all participants are given. For CGF this measure is not applicable as it guarantees error values below a given tolerance margin. 


\subsection{Limitations}

We used glasses to attach the vibration motors to the user. This provides good contact to the mastoid bone to conduct vibrations. In a later HI application the fitting might be not that tight requiring higher vibration intensities.

Moreover, we focused on the feedback component of the overall system. We assume the feasibility of calculating the target angle of relevant sound sources occurring in daily life situations. To assess the relevance of sound sources, context information needs to be considered, e.g. by context recognition based on HI-BANs.

To show the effective benefit of the system, a larger user study with hearing impaired participants that rate the complete processing chain in mobile daily life settings would be required. However, as a first step our focus in this explorative work was to systematically evaluate vibrotactile encoding schemes for lateralization to assess the potential benefit.

\section{CONCLUSION AND OUTLOOK}

Our results encourage to use bilateral vibrotactile feedback to support localization of sound sources, especially for users of HIs and cochlear implants that have difficulties with lateralization. Results show a trade off between user response time and minimum angular lateralization error. Choosing the most suitable bilateral vibrotactile encoding schemes depends on the requirements of the application: Bilateral vibrotactile encoding can be optimized for low RMS lateralization error or short response time while considering the available energy budget. For QAH encoding schemes results also show a trade off between the minimal quantization error due to vibrotactile encoding and the average number of user errors due to misinterpretation of presented patterns. The simpler the schemes are concerning the number of encoded segments, the lower are the user error and response time, but the higher the induced quantization error due to vibrotactile encoding. For the complex encoding schemes the overall performance of the vibrotactile feedback for lateralization is person-specific. The presented results are applicable to other domains of human computer interaction such as navigation or gaming.

We plan to deploy the system in a study with hearing impaired people to evaluate the benefit of the system with combined acoustic and vibrotactile feedback.

\section{Acknowledgements}

This work was part funded by CTI project 10698.1 PFLS-LS "Context Recognition for Hearing Instruments Using Additional Sensor Modalities". The authors gratefully thank all participants of the experiment.

\section{REFERENCES}

[1] T. Ahmaniemi and V. Lantz. Augmented reality target finding based on tactile cues. In International conference on Multimodal interfaces, 2009.

[2] A. Biggins. Benefits of wireless technology. Hearing Review, 112009.

[3] E. Borg, J. Ronnberg, L. Neovius, and T. Lie. Vibratory-coded directional analysis: Evaluation of a three-microphone/four-vibrator DSP system. J. of rehabilitation research and development, 38(2), 2001.
[4] J. Craig. Difference threshold for intensity of tactile stimuli. Attention, Perception, $\&$ Psychophysics, 11(2):150-152, 1972.

[5] G. Gescheider. Temporal relations in cutaneous stimulation. Cutaneous communication systems and devices by F.A. Geldard. Austin, Tex.: The Psychonomic Society, 1974.

[6] S. Kochkin. MarkeTrak VIII: 25-year trends in the hearing health market. Hearing Review, 2009.

[7] J. Loomis, R. Golledge, and R. Klatzky. Navigation system for the blind: Auditory display modes and guidance. Presence, 7(2):193-203, 1998.

[8] M. Mueller et. al. Localization of virtual sound sources with bilateral hearing aids in realistic acoustical scenes. PACS, 2011.

[9] K. Myles. Guidelines for Head Tactile Communication. Technical report, Army Research Lab Aberdeen Proving Ground Md Human Research And Engineering Directorate, 2010.

[10] L. Petrosino and D. Fucci. Temporal resolution of the aging tactile sensory system. Perceptual and motor skills, 1989.

[11] M. Pielot, B. Poppinga, and S. Boll. PocketNavigator: vibro-tactile waypoint navigation for everyday mobile devices. In Conference on Human computer interaction with mobile devices and services, 2010.

[12] B. Schmalz. USB Bit Whacker - An inexpensive, simple input/output device to connect your computer to the real world, 2011.

[13] J. Shargorodsky, S. Curhan, G. Curhan, and R. Eavey. Change in Prevalence of Hearing Loss in US Adolescents. JAMA, 304(7):772, 2010.

[14] M. Straub, A. Riener, and A. Ferscha. Route guidance with a vibro-tactile waist belt. In 4 th European Conference on Smart Sensing and Context, 2009.

[15] B. Tessendorf, A. Bulling, D. Roggen, T. Stiefmeier, M. Feilner, P. Derleth, and G. Tröster. Recognition of hearing needs from body and eye movements to improve hearing instruments. In International Conference on Pervasive Computing, 2011.

[16] B. Tessendorf et. al. Design of a bilateral vibrotactile feedback system for lateralization. In International ACM SIGACCESS Conference on Computers and Accessibility (ASSETS'11), 2011.

[17] J. van Erp. Guidelines for the use of vibro-tactile displays in human computer interaction. In Proceedings of Eurohaptics, volume 2002, 2002.

[18] J. Weisenberger, A. Heidbreder, and J. Miller. Development and preliminary evaluation of an earmold sound-to-tactile aid for the hearing-impaired. J Rehabil Res Dev, 24:51-66, 1987. 